\title{
A Tutorial About Tower Transmission Models for the Analyses and Prediction of Backflashovers
}

\author{
A. R. J. Araujo and S. Kurokawa
}

\begin{abstract}
Lightning is one of a main causes of sudden outages in the power systems. When a lightning strikes the ground wires or directly to transmission tower, a backflashover may occur. It consists of an electric arc from the tower structure to the line phase conductors, causing transient voltage surges in the transmission line and may cause an outage. In this context, an electric parameter named tower surge impedance plays a key role for the prediction and estimation of backflashovers. Several authors have proposed models representing the transmission tower in order to estimate its surge impedance and measure overvoltages caused by the lightning on transmission lines. In this article, a brief historical survey on transmission towers modelling proposed by diverse authors, a description of the backflashover and the most common models for representing towers are presented.
\end{abstract}

Keywords - backflashover, tower modeling, transmission towers, overvoltage.

\section{INTRODUÇÃO}

A s descargas atmosféricas são uma das principais fontes de distúrbios causados em linhas de transmissão no Brasil, gerando sobretensões nas linhas de transmissão que podem levá-las ao seu desligamento. As faltas causadas por descargas atmosféricas causam sérios danos nas linhas de transmissão, cadeias de isoladores, problemas de estabilidade e blackouts [1]. Nesse intuito, análises de descargas atmosféricas sobre torres das linhas de transmissão desempenham um papel fundamental na sua proteção. Quando a descarga atmosférica atinge diretamente o cabo-guarda ou a torre, a intensidade do campo elétrico em torno da estrutura da torre se eleva significativamente. A partir de um dado instante, o campo elétrico em torno da estrutura atinge um valor crítico, causando o rompimento da rigidez dielétrica do ar. Um arco elétrico é gerado, contornando a cadeia de isoladores, entre a estrutura da torre e os condutores de fase, denominado de backflashover. Os backflashovers exercem importante influência no desempenho de um sistema de transmissão, pois estima-se que entre $40 \%$ a $70 \%$ dos desligamentos de linhas de transmissão são provocadas por esse fenômeno [2]. Esses incidentes ocorrem principalmente em regiões com alta incidência de descargas atmosféricas, grande resistividade do solo e relevo elevado [2]. Para reduzir a ocorrência de backflashovers, é necessário a instalação de cabos-guarda, o dimensionamento correto da torre de transmissão e da cadeia

A. R. J. Araujo, Universidade Estadual Paulista (UNESP), Faculdade de Engenharia de Ilha Solteira, Ilha Solteira, Brasil, anderjusto@yahoo.com.br

S. Kurokawa, Universidade Estadual Paulista (UNESP), Faculdade de Engenharia de Ilha Solteira, Ilha Solteira, Brasil, kurokawa@dee.feis.unesp.br de isoladores e uma pequena impedância de aterramento. As sobretensões dependem da impedância de surto da torre, diretamente ligada com a geometria da torre, da forma de onda da corrente injetada no topo da torre e da impedância de aterramento [3]-[5]. A impedância de surto pode ser calculada por: medições na torre de transmissão de tamanho real ou em modelos de escala reduzida, modelos geométricos e por métodos computacionais [4], [6], [7]. Diversos autores realizaram medições da impedância de surto e da velocidade de propagação diretamente em torres reais ou em modelos em escala reduzidas [1], [5], [8]. Em torres reais são utilizados geradores de pulso, suspensos por balões, que injetam correntes no topo da torre de transmissão [1],[4],[9]. Esse método é eficiente, porém caro e de difícil implementação. Uma alternativa são os modelos em escala-reduzida que fornecem uma boa estimativa para a impedância de surto, porém problemas com interferência eletromagnética que requerem muita atenção durante as medições [5], [8].

A impedância de surto da torre pode ser estimada por modelos geométricos. Nesse caso, a torre é aproximada por sólidos simples tais como cones e cilindros, ou por uma combinação desses. A partir dessa representação, equações específicas foram desenvolvidas para cada formato de torre [3],[7],[9]. Entretanto, essas equações não consideram os braços, as barras diagonais (treliças) da estrutura, impedância de aterramento da torre de transmissão e o tipo de solo em questão. $\mathrm{O}$ comportamento elétrico de uma torre de transmissão frente a uma descarga atmosférica depende de diversos fatores, tais como: a geometria da torre, impedância de surto da torre, impedância de aterramento e a forma e a direção de onda da corrente injetada na torre [5], [11], [12]. Assim, a impedância de surto desempenha um papel fundamental na determinação do potencial elétrico na torre, especialmente quando há possibilidade de ocorrer backflashovers sobre as cadeias de isoladores. Diversos modelos de torres de transmissão têm sido propostos por pesquisadores para a determinação das sobretensões causadas no sistema elétrico devido a descargas atmosféricas. Os modelos de torres são muito úteis na avaliação dos níveis de sobretensões e previsão de desligamentos devido a incidência de descargas atmosféricas. O conhecimento dos níveis de sobretensões permite que a especificação correta da cadeia de isoladores e o dimensionamento adequado da impedância de aterramento, reduzindo-se a possibilidade de backflashovers e aumento na confiabilidade do sistema elétrico.

Os objetivos deste trabalho consistem num levantamento histórico dos modelos de torres de transmissão, uma descrição do fenômeno backflashover e apresentação de alguns modelos 
que são propostos para representação das torres de transmissão e uma breve análise comparativa entre eles.

\section{LEVANTAMENTO HISTÓRICO DOS MODELOS DE TORRES DE TRANSMISSÃO}

Quando uma descarga atmosférica atinge diretamente o cabo-guarda ou a torre de transmissão, ondas viajantes de corrente percorrem repetidamente a estrutura da torre. Essas ondas são refletidas pela impedância de aterramento da torre e pelo topo da torre, elevando o potencial elétrico ao longo de seu comprimento e nos braços das torres. A diferença de tensão entre o condutor de fase e a estrutura da torre podem exceder o limiar crítico de flashover- CFO (Critical Flashover Voltage) produzindo um arco elétrico que contorna a cadeia de isoladores, se originando da torre para os condutores de fase, conforme a Fig. 1-(a) [13]. A cadeia de isoladores é submetida a uma onda de tensão cuja amplitude pode provocar a falha no isolamento e estabelecer um curto-circuito entre a fase e o torre, causando sobretensões na linha de transmissão Fig. 1-(b) [11],[14].

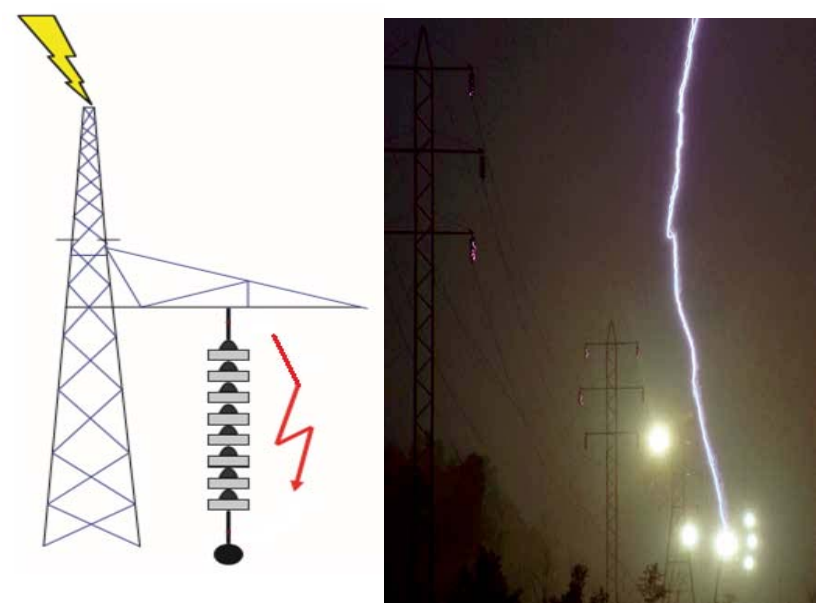

Figura 1. (a) Ilustração de um backflashover (b) Backflashover em torre real.

Diversos estudos têm sido realizados para avaliar as sobretensões causadas pelas descargas atmosféricas e estimar a impedância de surto da torre [9]-[12]. Assim alguns modelos para as torres de transmissão foram desenvolvidos no domínio da frequência ou diretamente no domínio do tempo desde a década de 1960, sendo empregados em programas como ATP, EMTP ou PSCAD.

A primeira formulação teórica para a impedância de surto da torre de transmissão foi proposta por [15]. Nessa formulação, assume-se que toda a corrente é uniformemente distribuída pela estrutura da torre, desde sua base ao topo. A estrutura da torre é aproximada por um cilindro vertical com altura equivalente à da torre e o raio é igual ao raio médio equivalente de sua base. A velocidade de propagação ao longo da torre é adotada como a velocidade da luz. Diversas formulações teóricas para o cálculo da impedância de surto usando a teoria de campo eletromagnético e geometria de sólidos simples foram propostas por [16-19]. Nesses artigos é levado em consideração o efeito do vetor potencial magnético gerado devido à injeção de corrente no topo da torre e a velocidade de propagação das ondas ao longo da torre é considerada a velocidade da luz.

O modelo geométrico consiste na representação da torre de transmissão por sólidos simples, tais como cilindros, cones ou por combinação desses sólidos [18]-[20]. Uma vez que a torre é aproximada por sólidos simples, equações para calcular a impedância de surto são deduzidas. Embora essas equações sejam atrativas e simples, o problema consiste em como representar adequadamente a torre de transmissão por esses sólidos. Assim a geometria da torre é aproximada superficialmente e braços da torre são desconsiderados, levando a erros no computo da impedância de surto.

As medições em torres em escala real foram realizadas por [5] e [12] usando método direto. Nesse método, o gerador de impulso é conectado ao topo da torre e cabos de injeção de corrente impulsiva, sustentados por balões, são posicionados de modo a variar o ângulo de incidência do pulso. As correntes que percorrem a estrutura da torre são medidas por cabos coaxais e transformadores de corrente instalados ao longo de sua estrutura. Diversos estudos foram conduzidos no Japão e os resultados medidos se tornaram uma base para simulações computacionais em programas de análises de transitórios [13]. Medições feitas com descargas elétricas reais ou induzidas por foguetes em torres de linhas de transmissão foram realizadas por [23]-[25]. Todos eles estimaram a impedância de surto da torre baseados nessas medições e propuseram um modelo de torre disponível no EMTP. Os resultados mostraram que a impedância de surto $\mathrm{e}$ as sobretensões de surto dependem da direção que a corrente è injetada no topo da torre de transmissão. Os autores também obtiveram êxito nas medições simultâneas de correntes em várias partes da torre de transmissão em teste, assim como as tensões sobre as cadeias de isoladores [23]-[25].

Métodos baseados em escala reduzida são também frequentemente empregados. Os modelos em escala reduzida são mais econômicos do que os modelos em torres do tamanho real, além de serem mais flexíveis e de fácil implementação [ 3], [9], [21]. As simulações conduzidas em estruturas de escala reduzida são dificilmente empregadas para cálculos práticos em linhas de transmissão, mas essa aproximação é útil para compreender o comportamento das descargas e os efeitos eletromagnéticos no regime transitório de um sistema tridimensional atingido pela descarga atmosférica. Outro fator importante seria a precisão na medição, especialmente quando o método de medição direto é aplicado. Nessa condição, o tamanho dos equipamentos (geradores de impulso e de medição) é relativamente grande quando comparados com a torre em escala reduzida [22].

Métodos de análises numéricas também têm sido aplicados para o cálculo da impedância de surto de torres [26], no qual métodos dos elementos finitos são usados nas simulações com EMTP. Em [5] os autores analisaram uma torre de transmissão em escala usando o Método dos Momentos (MoM), incluindo toda a estrutura da torre. Em [27], o Método das Diferenças Finitas no Domínio do Tempo (FDTD) é aplicado para simular a resposta a um degrau de tensão em uma torre de transmissão de $500 \mathrm{kV}$, incluindo a 
resistividade do solo e estrutura completa da torre. A seguir, alguns modelos de torres de transmissão são apresentados.

\section{MODELOS DE TORRES DE TRANSMISSÃO}

No desenvolvimento dos modelos das torres de linhas de transmissão, diversos autores propuseram modelos que são classificados como:

\author{
i. Modelos geométricos; \\ ii. Modelos multicondutores; \\ iii. Modelos "multi-andares"; \\ iv. Modelos dependentes da frequência.
}

\section{A. Modelos geométricos}

Os primeiros modelos de torres de transmissão foram desenvolvidos utilizando a teoria de campo eletromagnético, representando a torre por geometria simples, tais como cilindros, cones ou combinação desses sólidos. Em [17] usa-se um modelo cilíndrico e a impedância de surto da torre depende da direção em que as ondas de corrente são injetadas no topo da torre. Em [18], representa-se a torre por um cone e uma alteração no modelo cilíndrico é proposta. Em [3] é proposta uma equação modificada para os modelos cilíndricos e cônicos, e recomenda-se um modelo para torres de transmissão estreita, sendo esse modelo também recomendado por CIGRE. Embora a velocidade de propagação ao longo da torre possa ser estabelecida como a velocidade da luz, os múltiplos caminhos da estrutura de treliças e os braços de torre inserem atrasos; assim o tempo para ondas de tensão e corrente refletirem do solo ao topo da torre é maior do que o tempo de viagem esperado. Em [3], [17] e [18] equações para o cálculo da impedância de surto da torre, considerando distintas formas geométricas e a orientação da injeção da corrente no topo da torre, são apresentadas. A Fig. 1 mostra a silhueta de uma torre de transmissão e aproximações por geometria simples.

$\mathrm{Na}$ Fig.2-(a) mostra a silhueta típica de uma torre de transmissão enquanto que Figs 2-(b) a (d) mostram as geometrias cilíndrica, cônica e mista (combinação) propostas por [3], [17] e [18]. As equações da impedância de surto das torres são apresentadas na tabela I. Na tabela $1, h$ e $r$ são a altura e o raio da base do cilindro ou cone. Na eq. (7), $r_{1}, r_{2}$ e $r_{3}$ são os raios do topo, do meio e da base da torre respectivamente e $h_{1}$ e $h_{2}$ as alturas mostradas na Fig. 2-(d).

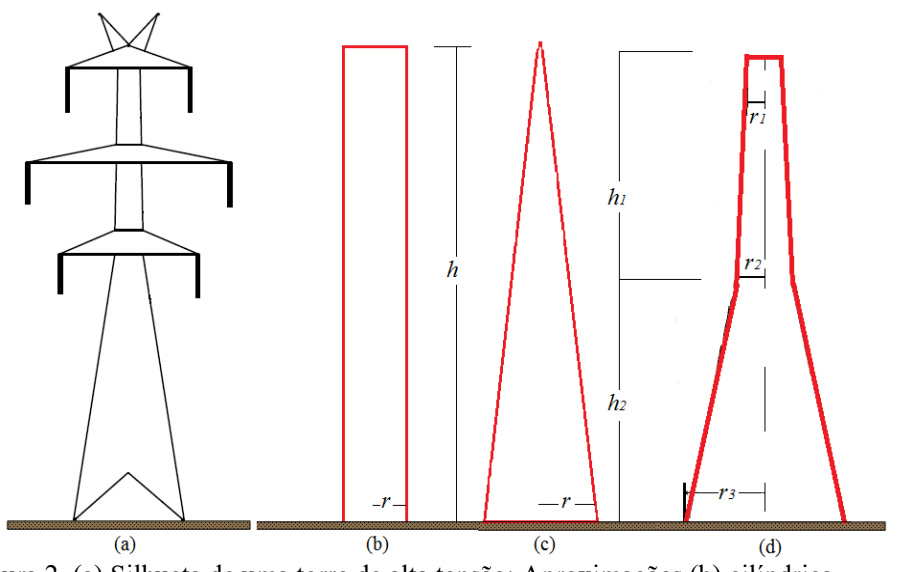

Figura 2. (a) Silhueta de uma torre de alta tensão; Aproximações (b) cilíndrica, (c) cônica e (d) por troncos de cone.

TABELA I

IMPEDÂNCIA DE SURTO DA TORRE PARA O MODELO APROXIMADO POR GEOMETRIA SIMPLES

\begin{tabular}{|c|c|c|}
\hline $\begin{array}{l}\text { REFERÊNCIA/ } \\
\text { APROXIMAÇÃO }\end{array}$ & IMPEDÂNCIA DE SURTO & $\begin{array}{l}\text { EQUAÇ } \\
\text { ÃO }\end{array}$ \\
\hline [3]-Cônica & $\mathrm{Z}_{\mathrm{T}}=60 \ln \left(\cot \left(\frac{1}{2} \tan ^{-1}\left(\frac{r}{h}\right.\right.\right.$ & (1) \\
\hline [3]-Cilíndrica & $\mathrm{Z}_{\mathrm{T}}=60 \ln \left(\cot \left(\frac{1}{2} \tan ^{-1}\left(\frac{r}{h}\right)\right.\right.$ & (2) \\
\hline $\begin{array}{l}\text { [3]-Comb. de } \\
\text { Sólidos }\end{array}$ & $\mathrm{Z}_{\mathrm{T}}=60 \ln \left(\cot \left(\frac{1}{2} \tan ^{-1} T\right.\right.$ & (3) \\
\hline $\begin{array}{l}{[17]-} \\
\text { Cilíndrica }\end{array}$ & $Z_{T}=60\left[\ln \left(\frac{2 \sqrt{2 h}}{r}\right)-2\right.$ & (4) \\
\hline $\begin{array}{c}\text { [18]- } \\
\text { Cilíndrica }\end{array}$ & $\mathrm{Z}_{\mathrm{T}}=60\left[\ln \left(\frac{2 \sqrt{2 h}}{r}\right)-1\right]$ & $(5)$ \\
\hline [18]- Cônica & $\mathrm{Z}_{\mathrm{T}}=60 \ln (\sqrt{2}$ & (6) \\
\hline
\end{tabular}

Onde $T$ é dado por:

$$
\mathrm{T}=\frac{r_{1} h_{2}+r_{2}\left(h_{1}+h_{2}\right)+r_{3} h_{1}}{\left(h_{1}+h_{2}\right)^{2}}
$$

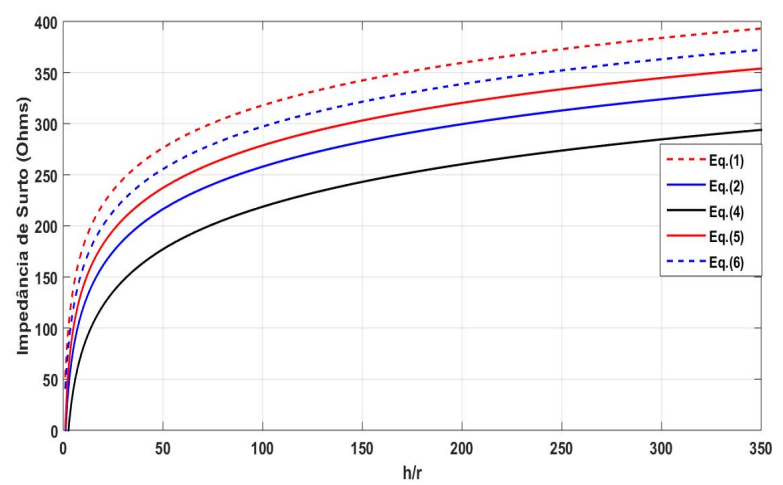

Figura 3. Impedância de surto para distintas equações da tab. 1, em função de $\mathrm{h} / \mathrm{r}$. 
A Fig. 3 mostra o comportamento da impedância de surto em função de $h / r$. Observa-se que para um $h / r$ fixo, a impedância de surto calculada pelas Eqs. (1) e (6), equações para a forma cônica, são superiores do que para equações na forma cilíndrica. Assim, erros podem ser inseridos nas análises de sobretensões dependendo do tipo de geometria aplicada. Esses modelos servem apenas como uma estimativa para calcular a impedância de surto da torre, porém modelos mais precisos devem ser usados para analises mais críticas. Em [35], os autores apresentam uma revisão na fórmula de Jordan, para o computo da impedância de surto de condutores verticais, e estendida para sistema multi-condutores.

\section{B. Modelos multicondutores}

Nesses modelos a torre de transmissão é dividida em 4 seções e cada uma é representada por linhas curtas sem perdas ou por um condutor de raio equivalente. A exata impedância de cada trecho é dada em função da geometria, baseadas em uma série de experimentos em modelos de escala reduzida. Cada trecho é representado por uma linha de transmissão monofásica com um determinado raio equivalente $[6,28]$. Em [6] obteve-se computacionalmente as formas de onda para as tensões nos braços da torre de circuito duplo independente. Essas tensões estão de acordo com as obtidas experimentalmente. Assim os autores propõem uma fórmula empírica para impedância de surto para um condutor vertical de cada trecho da torre. A Fig. 4 mostra os modelos por linhas sem perdas.

Na Fig. 4-(b) a torre de transmissão é dividida em quatro partes, na qual cada parte é representada por um condutor cilíndrico de raio equivalente. Na Fig.4-(c) mostra as impedâncias de surto $Z_{T i}$ para $(i=1, \ldots, 4)$ para cada trecho de torre. Em [1], os autores modificam o modelo de multicondutores, incluindo o efeito das barras diagonais entre os condutores verticais da torre (representado por linhas sem perdas em paralelo com as pernas da torre- $Z_{L i}$ ) e os braços (representadas como linhas sem perdas ramificadas a partir do seu ponto de junção com a estrutura da torre- $Z_{A i}$ ), conforme a Fig. 4-(d)

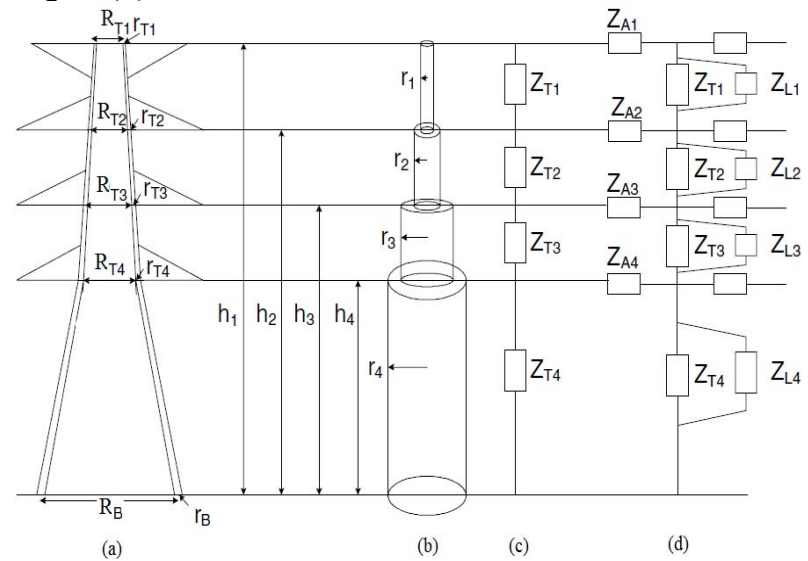

Figura 4. (a) Silhueta de uma torre; (b) Condutores de raio equivalente para cada trecho; (c) Modelo simples; (d) Modelo incluindo treliças e braços de torre.

Resultados teóricos mostram que a impedância de surto dos condutores é reduzida em $10 \%$ com adição das barras diagonais entre as pernas principais da torre. Em [1] apresenta-se as fórmulas empíricas para $r_{i}, Z_{T i}, Z_{A i}$ e $Z_{L i}$.

\section{Modelos "multi-andares"}

No modelo "multi-andares", a torre é dividida em 4 seções na qual cada seção consiste de uma linha de transmissão sem perdas em série $\mathrm{Z}_{\mathrm{T}}$ com um bloco $\mathrm{R}-L$ paralelo. Esses blocos consideram o efeito da atenuação das ondas viajantes ao longo dos condutores. Nesse modelo, a resistência representa a atenuação da onda de tensão que viaja pela torre e a indutância em paralelo faz com que a resistência gradualmente se torne ineficiente à medida que o tempo passa. Esse modelo pode ser interfaceado facilmente no EMTP ou ATP, sendo apropriado para análises com linhas de multicondutores. Os parâmetros da torre são obtidos experimentalmente em linhas de alta tensão de circuito simples ou duplo com cabos guarda [9]. Em [25], os parâmetros são obtidos para uma torre de transmissão de 275 $\mathrm{kV}$ com cabos guarda e circuito duplo atingida por descargas atmosféricas naturalmente ou induzidas por foguetes em dias de tempestade.

Em geral, uma torre de transmissão de alta tensão pode ser representada pelo modelo "multi-andares" conforme a Fig. 5. Diversos autores propõem os parâmetros $R_{i}, L_{i}$ e $Z_{T i}$ para cada seção da torre [9],[12],[25],[30]. A corrente de descarga no topo da torre e as correntes que percorrem os cabos-guarda são medidas por conectores coaxiais resistivos e as correntes que fluem pelas "pernas" das torres são medidas por transformadores de corrente. As tensões elétricas nas cadeias de isoladores são medidas por divisores resistivos de tensão. Embora os parâmetros necessitem de mais investigações, o modelo "multi-andares" pode ser usado para as análises das sobretensões em linhas de transmissão com boa precisão [25],[30]. Os parâmetros foram deduzidos a partir de medições experimentais sendo que em [29] propõe-se uma revisão no modelo e em seus parâmetros no seu artigo.

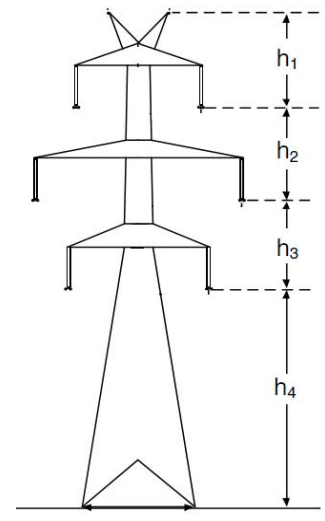

(a)

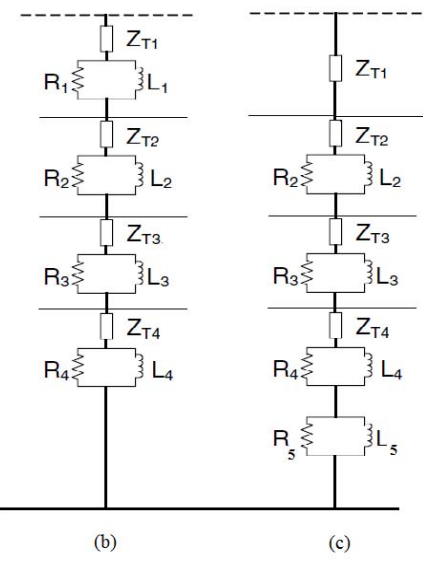

c)
Figura 5. (a) Silhueta típica de uma torre de alta tensão; (b) Modelo "multiandares" proposto por [9], [12]e [25];(c) modelo proposto por [31].

Em [30] apresenta-se as simulações de descargas atmosféricas que atingem linhas de transmissão de $150 \mathrm{kV}$ e $400 \mathrm{kV}$, estimando a corrente mínima para provocar os backflashovers, levando em consideração a resistência de aterramento da torre. Foram utilizados os modelos geométrico (i), modelos compostos por linhas sem perdas (ii) e modelos 
"multi-andares" (iii) onde são apresentados os valores $R_{i}, L_{i} \mathrm{e}$ $Z_{T i}$ para linhas de $150 \mathrm{kV}$ e $440 \mathrm{kV}$. O modelo "multi-andares" é de fácil implementação em software de análises de transitórios eletromagnéticos em sistemas de potências tais como ATP, EMTP e PSCAD. Um estudo apresentado [31] mostra que esse modelo não é adequado para representação de torres de baixa tensão, sendo que para torres de transmissão curtas podem-se utilizar modelos mais simples, tais como quatro linhas sem perdas representando a torre de maneira satisfatória.

\section{Modelos dependentes da frequência}

Os modelos dependentes da frequência representam a torre de transmissão por linha de transmissão no domínio da frequência para simular os transitórios eletromagnéticos, considerando a distorção das ondas viajantes. Em [32] e [33], a torre de transmissão é representada por uma linha uniforme, como uma impedância de surto dependente da frequência, sendo interfaceado pelo EMTP. Em [12] apresenta-se uma aproximação para o cálculo da impedância de surto da torre baseado na representação da torre por uma linha de transmissão. Em [12], as correntes e tensões são computadas nos terminais emissor e receptor de uma linha equivalente, determinadas por métodos numéricos implementados no domínio da frequência. Para esse cálculo, a torre de transmissão é representada por um quadripolo e as correntes e tensões são descritas pelas equações hiperbólicas da linha no domínio da frequência. A representação da torre pela teoria de quadripolos pode ser aplicada a qualquer geometria para o cálculo da impedância de torre
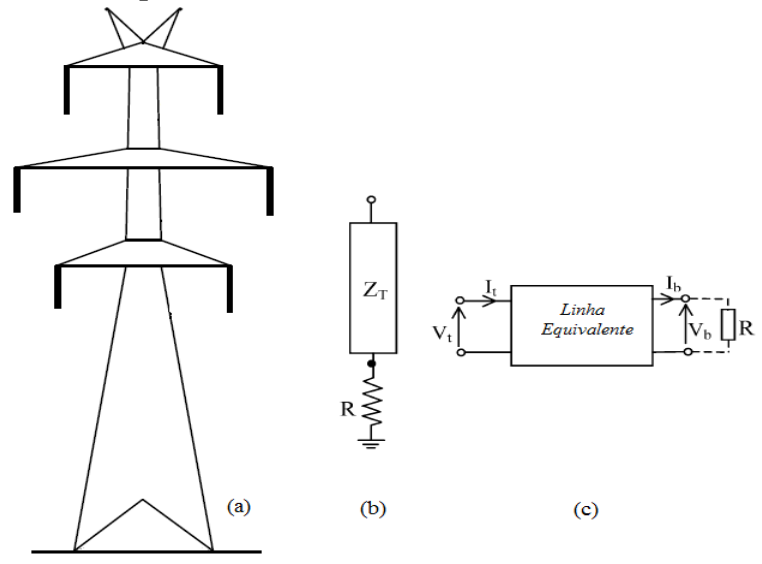

Figura 6. (a) Torre de transmissão para alta tensão. (b) representação da torre como uma linha de transmissão. (c) torre de transmissão representada por quadripolo.

A Fig. 6-(a) mostra uma torre de transmissão de alta tensão genérica que será representada por uma linha de transmissão vertical (Fig. 6-(b)) e representada por um quadripolo (Fig. 6(c)). Em [12] é proposto um método para o cálculo da impedância de surto da torre, no domínio da frequência, utilizando medições das correntes e tensões nos terminais emissor e receptor da torre, considerando o solo com distintas resistividades. Em [12] também é apresentado diversos métodos para medições da impedância de surto no domínio do tempo. Uma das equações sugeridas é dada por (8) [18]:

$$
\mathrm{Z}=\frac{\mathrm{V}_{P}}{\mathrm{I}_{\mathrm{V}_{\mathrm{p}}}}
$$

Em (8), $V_{p}$ é a tensão instantânea de pico e $I_{V_{p}}$ é corrente no instante do pico da tensão, sendo esse método válido para aproximação de ondas viajantes, considerando as primeiras reflexões [12]. A tabela II mostra a impedância de surto calculada para torres de $275 \mathrm{kV}$ e $400 \mathrm{kV}$, usando as equações do modelo geométrico [18] e [34], o método de medições no domínio do tempo [12]* e dos quadripolos [12]**, conforme mostrado.

TABELA II

COMPARAÇÃO DA IMPEDÂNCIA DE SURTO CALCULADA POR DIVERSOS MÉTODOS

\begin{tabular}{ccccc} 
TORRE $(\mathrm{KV})$ & {$[12]^{*}$} & {$[12]^{* *}$} & {$[18]$} & {$[34]$} \\
\hline 275 & $182 \Omega$ & $189 \Omega$ & $176 \Omega$ & $196 \Omega$ \\
400 & $170 \Omega$ & $178 \Omega$ & $159 \Omega$ & $180 \Omega$ \\
\hline equação (8) **método proposto & & &
\end{tabular}

Assim esse método de quadripolos na frequência apresenta valores próximos aos demais métodos estudados em [12], sendo uma boa estimativa para cálculo e estimação da impedância de surto de torres de transmissão.

\section{CONCLUSÕES}

Os modelos de torres de transmissão têm sidos propostos por diversos autores e foram apresentados sumariamente neste artigo. O levantamento histórico mostrou a importância que os pesquisadores têm dedicado ao estudo das sobretensões causadas por backflashover. Os modelos geométricos (i), aproximam as torres por geometrias simples para o cálculo da impedância de surto. Apesar de ser de fácil uso, não é tão preciso, pois desconsidera partes da estrutura da torre, treliças e a resistividade do solo. Outra característica importante é que dependem da direção da corrente injetada. Os modelos multicondutores e "multi-andares" de torres (ii, iii) levam em conta importantes características das torres, tais como sua geometria e treliças, e representam a torre por meio de parâmetros discretos de circuitos elétricos (para computar o efeito do amortecimento das ondas viajantes ao longo de sua estrutura). Além disso, esses modelos podem ser utilizados diretamente no domínio do tempo e ou inseridos nos softwares para estudos de transitórios eletromagnéticos tais como EMTP, ATP e PSCAD. O modelo dependente da frequência (iv) apresenta um modo interessante para estimar a impedância de surto. Assim uma torre de transmissão é vista como uma linha de transmissão no domínio da frequência e são empregadas as equações hiperbólicas da linha. Também são apresentados métodos no domínio do tempo, nos quais apresentam valores próximos aos obtidos para a torre de transmissão aproximada por geometria simples (tabela 2). Os diversos modelos propostos mostram a importância da estimação da impedância de surto das torres de transmissão na previsão de sobretensões causadas por descargas atmosféricas e na sua prevenção. 


\section{AGRADECIMENTOS}

Esta pesquisa é fomentada em partes pela Fundação de Amparo à Pesquisa do Estado de São Paulo (FAPESP), Projeto de doutorado 2014/18551-6 e pelo Conselho Nacional de Desenvolvimento Científico e Tecnológico (CNPq).

\section{REFERÊNCIAS BIBLIOGRÁFICAS}

[1] T. Hara; O. Yamamoto, "Modeling of a transmission tower for lightningsurge analysis", IEE Proc. Generation, Transmission and Distribution, vol. 143, no 3, pp.283-289, Maio 1996.

[2] W. Shuwen; S. Wenxin, "Backflashover protection performance analysis of $220 \mathrm{kV}$ double circuit transmission line", Proc. Power and Energy Engineering Conferece (APPEEC), Wuhan, China, Março 2011.

[3] W. A, Chisholm; Y. L, Chow; K. D. Srivastava, "Lightning Surge Response of Transmission Towers," IEEE Trans., vol. PAS-102, pp.3232-3242, 1983.

[4] H. Motoyama; H. Matsubara."Analytical and experimental study on surge response of transmission tower", IEEE Transactions on Power Delivery, vol. 15, no 2, pp. 812-819, abril, 2000.

[5] Y. Baba; M. Ishii, "Numerical electromagnetic field analysis on measuring methods of tower surge impedance", IEEE Transactions on Power Delivery, vol. 14, no 2, pp.630-635, abril, 1999.

[6] M. Chanaka; K. Shanthi; R. Perera, "Modeling of power transmission lines for lightning back flashover analysis (A case study: $220 \mathrm{kV}$ Biyagama-Kotmale transmission line), Proc. 6th International Conference on Industrial and Information Systems (ICIIS 2011), Peradeniya, Sri Lanka, agosto 2011.

[7] T. Cao; T. Pham; S. Boggs, "Computation of tower surge impedance in transmission line", Eletrical Insulation Conference, Ottawa, Canadá, 2 a 5 junho, 2013.

[8] J. Takami; T. Tsuboi; K. Yamamoto et al, "Lightning surge response of a double-circuit transmission tower with incoming lines to a substation through FDTD simulation", IEEE Transactions on Dielectrics and Electrical Insulation, vol. 21, no 1, pp. 96-104, fevereiro, 2014.

[9] M. Ishii; T. Kawamura; T. Kouno, "Multistory transmission tower model for lightning surge analysis", IEEE Transactions on Power Delivery, vol. 6, no 3, pp.1327-1335, julho, 1991.

[10] Z. Zakaria; S. Bashi; N. F. Mailah et al, "Simulation of lightning surges on tower transmission using PSCAD/EMTDC: A comparativestudy", Proc. 2002 Student Conference on Research and Development, Shah Alam, Malaysia, julho 2002.

[11] P. Yadee; S. Premrudeepreechacharn, "Analysis of Tower Footing Resistance Effected Back Flashover Across Insulator in a Transmission System", In: IPST-International Conference on System Transients, Lyon, França,2007.

[12] N. Harid, N.; Griffiths, H.; Haddad, A, "A New Frequency-Dependent Surge Impedance Calculation Method for High-Voltage Towers", IEEE Transaction on Power Delivery, vol. 21, no 3, pp. 1430-1437, julho 2006.

[13] S. A Halin ; A. H. A. Bakar; H. A. Illias. et al. "Lightning backflashover tripping patterns on a $275 / 132 \mathrm{kV}$ quadrupule circuit transmission line in Malaysia" IET Science, Measurment and Tecnology, vol. 10, n. 4, pp. 344-354, Dezembro 2015.

[14] O. E. Gouda, A. Z. El Dein, G. M. Amer "Parameters Affecting the Back Flashover across the Overhead Transmission Line Insulator Caused by Lightning ", Proceedings of the 14th International Middle East Power Systems Conference (MEPCON'10), Cairo University, Paper ID 111, Egito, dezembro 19-21, 2010.

[15] C. A. Jordan, "Lightning Computations for Transmission Lines with Overhead Ground Wires Part II," General Electric Review, vol. 34, pp. 180-185, 1934.

[16] R. Lundholm; R. B. Finn; W. S. Price, "Calculation of Transmission Line Lightning Voltage by Field Concepts," AIEE Transactions, vol. 76, pp. 1271-1283, 1958.

[17] C. F. Wagner; A. R Hileman, "A New Approach to Calculation of Lightning Performance of Transmission Lines (part II, III)," AIEE Trans., pt. III, vol. 78/79, pp. 996-1021/589-603, 1960.

[18] A. Sargent; M. Darveniza, "Tower Surge Impedance", IEEE Transactions., vol. PAS-88, pp. 680-687, 1969.
[19] K. Okumura; A. Kijima, "A Method for Computing Surge Impedance of Transmission Line Tower by Electromagnetic Field Theory," IEE of Japan, Trans. B, vol. 105, pp. 733-740, 1985.

[20] T. Cao; T. Pham; S. Boggs, "Computation of tower surge impedance in transmission line", Eletrical Insulation Conference, Ottawa, Canadá, 2 a 5 junho, 2013.

[21] A. A. M. WAHAB; I. MATSUBARA;H. KINOSHITA, “An Experimental Evaluation of Some Factors Affecting Tower Surge Impedance," Trans.IEE of Japan, vol. 107, pp. 171-177, 1987

[22] A. J. G. PINTO, "Modelagem de torres de transmissão utilizando análise numérica dos campos eletromagnéticos e funções racionais",27/02/2014, $124 \mathrm{f}$. Tese (Doutorado em Engenharia Elétrica)-Universidade Estadual de Campinas, UNICAMP, Campinas, 2014.

[23] Y. Matsumoto; O. Sakuma; K. Shinjo et al, "Measuring of Lightning Surges on Test Transmission Line Equipped with Arresters Struck by Natural and Triggered Lightning," IEEE Trans., PWRD, vol. 11, pp. 996-1002, 1995.

[24] K. Shinjo; Matsumoto,Y.;Sakuma, O. et al.“Characteristics of Transient Response of Okushishiku Test Transmission Line Struck by Natural and Triggered Lightning," Trans. IEE of Japan, vol. 117-B, pp. 478-487, 1997.

[25] H. Motoyama; K. Shinjo; Y. Matsumoto et al. "Observation and Analysis of Multiphase Back Flashover on the Okushishiku Test Transmission Line caused by Winter Lightning," in IEEE PES 1998 Winter Meeting, 1997, PE-204-PWRD-0-12-1997

[26] M. E. ALMEIDA; M. T. C. BARROS, "Tower modeling for lightning surge analysis using Electro-Magnetic Transient Program”, IEE Proc.Gener, Transm. Distrib., vol. 141, no.6, novembro, 1994.

[27] T. Noda, "A Numerical Simulation of Transient Eletromagnetic Fields for Obtaining the Step Response of a Transmission Tower Using the FDTD Method" IEEE Transactions on Power Delivery, vol. 23,n.2, pp. $1262-1263$

[28] J. A. Martinez; F. Castro-Aranda. "Tower Modeling for Lightning Analysis of Overhead Transmission Lines"Power Engineering Society General Meeting, vol.2, pp.1212-1217, junho 2005.

[29] Y. Baba; M. Ishii, "Numerical Electromagnetic Field analysis on Lightining Surge Response of Towerwith Shield Wire",IEEE Transactions on Power Delivery, vol. 15, no.3, pp.1010-1015, julho 2000.

[30] Z. Datsios; P. N Mikropolos, "Effect of tower modeling on the minimum backflashover current of overhead transmission lines", Conference: 19th International Symposium on High Voltage Engineering, Pilsen, Republica Tcheca.

[31] T, Ito; T. Ueda; H. Watanabe; T. Funabashi et al. "Lightning flashover on 77-kV systems: Observed voltage bias effect and analysis", IEEE Transactions on Power Delivery, vol. 18, n.2, pp 545-550, abril 2003.

[32] N. Nagaoka, "A development of frequeucy-dependent tower model", Transactions IEE Japan, vol. 111-B, no. 1, pp. 51-56, janeiro. 1991.

[33] S. Kato; A. Mochizuki; E. Zaima, "Transmission line models of tower in frequency domain" (in Japanese), Trans. IEE Japan, vol. 115-B, no. 5, pp. 524-531, maio 1995.

[34] E. C. Jordan and K. G. Balmain, Electromagnetic Waves and Radiating Systems, 2nd ed. Englewood Cliffs, NJ: Prentice-Hall, 1968, pp. 384396.

[35] A. De Conti, S. Visacro, A. Soares, and M. A. O. Schroeder, "Revision, extension and validation of Jordan's formula to calculate the surge impedance of vertical conductors," IEEE Trans. Electromagn. Compat., vol. 48, n. 3, pp. 530-536, Aug. 2006.

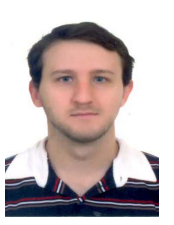

Anderson Ricardo Justo de Araújo graduado (2012) mestre (2014) em Engenharia Elétrica pela Universidade Estadual Paulista (UNESP), Faculdade de Engenharia de Ilha Solteira e doutorando na mesma instituição. Realiza um estágio de pesquisa com a bolsa BEPE/Fapesp (2016/091390) na Universidade de Manitoba (UofM), Canadá. Suas áreas de interesse são: transitórios eletromagnéticos em sistemas elétricos de potência utilizando métodos numéricos e modelagem de linhas /torres de transmissão.

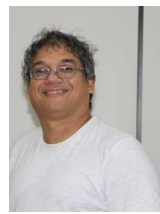

Sérgio Kurokawa (S'01-M'04) graduado em Engenharia Elétrica (1990). Desde 1994 atua como professor e pesquisador na Universidade Estadual Paulista (UNESP), Faculdade de Engenharia de Ilha Solteira. Obteve o título de 
Doutor em Engenharia Elétrica na Faculdade de Engenharia Elétrica e da Computação da Universidade Estadual Paulista (UNICAMP). Suas principais áreas de interesse são transitórios eletromagnéticos em sistemas elétricos de potência e modelos de linhas de transmissão para simulações de transitórios eletromagnéticos em sistemas de potência. 